\title{
Efficient direction of arrival estimation based on sparse covariance fitting criterion with modeling mismatch ${ }^{\text {光 }}$
}

\author{
Shu Cai ${ }^{a, *}$, Gang Wang ${ }^{b}$, Jun Zhang ${ }^{a}$, Kai-Kit Wong ${ }^{c}$, Hongbo Zhu ${ }^{a}$ \\ ${ }^{a}$ Jiangsu Key Laboratory of Wireless Communication, Nanjing University of Posts and \\ Telecommunications, Nanjing, China \\ ${ }^{b}$ Faculty of Electrical Engineering and Computer Science, Ningbo University, Ningbo, China \\ ${ }^{c}$ Department of Electronic and Electrical Engineering, University College London, London, \\ WC1E $7 J E, U K$
}

\begin{abstract}
This paper studies direction of arrival (DoA) estimation with an antenna array using sparse signal reconstruction (SSR). Among the existing SSR methods, the sparse covariance fitting based algorithms, which can estimate source power and noise variance naturally, are most promising. Nevertheless, they are either on-grid model based methods whose performance are sensitive to off-grid DoAs or gridless methods which are computationally demanding. In this paper, we propose an off-grid DoA estimation algorithm based on the sparse covariance fitting criterion. We first consider a scenario in which the number of snapshots is larger than the array size. An algorithm is proposed by applying an offgrid model, which takes into account the deviations between the discretized sampling grid and the true DoAs, to the sparse covariance fitting criterion. It estimates the on-grid parameters and the deviations of off-grid DoAs separately and thus is computationally efficient to implement. Then in the case where the number of snapshots is smaller than the array size, we propose to execute the

\footnotetext{
This work was partially supported by the National Natural Science Foundation of China (61427801, 61401231, and 61401232), the Natural Science Foundation of Jiangsu Province and NY214123), and Natural Science Foundation of the Higher Education Institutions of Jiangsu Province(15KJB510024).

${ }^{*}$ Corresponding author

Email address: caishu@njupt.edu.cn (Shu Cai)
} (BK20140873), Nanjing University of Posts and Telecommunications Program (NY213064
\end{abstract}

Preprint submitted to Signal Processing

February 1, 2017 
DoA estimation algorithm iteratively under the stochastic maximum likelihood (SML) criterion. The estimation accuracy and computational efficiency of the proposed algorithms are demonstrated by computer simulations.

Keywords:

DoA estimation, sparse parameter estimation, off-grid model, sparse covariance fitting.

\section{Introduction}

Finding directions of far-field narrowband signal sources based on observations of an antenna array is a classical problem in array signal processing [1]. In the past two decades, many high resolution direction of arrival (DoA) estimation methods have been proposed in the literature. The maximum likelihood (ML) principle is perhaps the most well-known statistically optimal criterion. However, solving the ML estimation problem directly is difficult in general since its objective function is nonlinear and nonconvex to the directions of interest. The Capon method [2] and subspace based algorithms [3, 4, 5, 6] estimate DoAs by studying the correlation matrix or its subspace. Although these methods are more efficient and can achieve the optimal estimation performance, they usually require a large number of snapshots and may experience a certain performance loss when dealing with highly-correlated sources. By studying the special structure of uniform linear arrays (ULAs), the algorithms, which are robust to signal correlations, such as the iterative quadratic maximum likelihood (IQML) method and the weighted subspace fitting (WSF) method, have been proposed (see [1] for a complete review).

Over the last decade, the developments in sparse signal reconstruction (SSR) provide a new perspective for DoA estimation. Roughly speaking, the SSR based DoA estimation methods can be divided into two classes: the on-grid model based method and the off-grid method. The on-grid model selects a fixed grid in the continuous direction range as the set of all possible DoA estimates and assumes that the true DoAs are exactly on the grid $[7,8,9,10]$. 
By employing $l_{1}$-norm to encourage sparsity and singular value decomposition (SVD) to reduce complexity, the $l_{1}$-SVD method [8] achieves superior resolution performance than many traditional methods. However, $l_{1}$-penalized estimators suffer from the problem of choosing thresholds for reconstruction error. By using a sparse covariance fitting criterion, the sparse iterative covariance-based estimation method (SPICE) avoids the issue of thresholds, and yet has good statistical properties [9]. In practice, the true DoAs usually do not satisfy the on-grid assumption, and thus the methods are only approximation methods. More specifically, a coarse grid leads to a high modeling error, whereas too dense a grid is computationally prohibitive (see [11] and reference therein).

Off-grid methods have emerged in order to alleviate modeling errors induced by the off-grid DoAs. The off-grid methods can be categorized into grid-based off-grid methods and gridless methods. In the first category, such as $[12,13,14,15,16,17,18,19]$, the errors between the true (off-grid) DoAs and grid points are parameterized and then estimated jointly with the on-grid unknown parameters. In the notable work of [12], Zhu et al. explored the structure of the basis matrix and proposed a sparse total least-squares approach to alleviate the effect of modeling inaccuracies. In [13], the authors presented a sparse spectral fitting with modeling uncertainty (SSFMU) estimator, which is based on convex relaxation and utilizes a diagonal loading approach to reduce the sensitivity to its optimization parameters. From a sparse Bayesian inference perspective, the authors of [19] proposed to iteratively recover the source signal and the matrix perturbation. However, to deal with the newly added off-grid related unknown parameters, existing off grid methods are usually more computationally expensive than the on-grid model based methods. Moreover, they are still grid based schemes whose performance depends on the tradeoff between computational complexity and grid size. Recently, a gridless sparse method was proposed by Candès and Fernandes-Granda to solve the problem of spectral analysis [20], which is mathematically equivalent to estimating DoAs based on a single-measurement-vector (SMV) of a ULA [21]. The method is then extended to the compressed sensing scenario [22] and the multiple-measurement-vectors 
(MMVs) case [23] via atomic norm minimization (ANM). On the other hand, in [11], Yang et al. proposed a sparse covariance fitting based gridless method, named as sparse and parametric approach (SPA), using convex optimization. An incomplete list of related literature includes [24, 25, 26, 27, 28, 29]. Besides having almost all the virtues belonging to the grid-based methods, the gridless methods completely avoid the off-grid problem and they are guaranteed to produce a sparse parameter estimation with a high probability under moderate conditions (see [22, 25] and [29] for example). However, these methods have only considered the linear array case. Recently, an interesting gridless method named as total variance minimization approach (TVMA) was proposed by Mahata et al., for arbitrary arrays [30]. Moreover, a gridless SPICE method was also obtained similarly. However, approximating an infinite series of JacobiAnger expansion by a finite series of Bessel function in TVMA also leads to modeling errors. On the other hand, to obtain an acceptable approximation, the order of Bessel functions required in the finite series is usually larger than the array size, which leads to more unknown parameters as well as a larger problem size than those of other gridless methods. Furthermore, the latter usually implies higher computational complexity.

In order to pursue a fast and accurate estimator whose application is not restricted by the array structure, we study the grid-based off-grid DoA estimation problem by incorporating off-grid error linearization given in [12] into the covariance fitting criterion [31]. However, the resulting problem of jointly estimating on-grid parameters and deviations of off-grid DoAs is nonlinear and nonconvex, and as a consequence very hard to solve. Our main contributions of this paper are summarized below.

1. A new algorithm named Capon-SPICE (C-SPICE) is proposed to solve the joint optimization problem in scenarios where the number of snapshots is greater than that of antennas. C-SPICE estimates the on-grid parameters and deviations of off-grid DoAs independently. When the deviations are found, the on-grid parameters can be updated iteratively in simple closed- 
forms and the iterations converge globally to an optimal point. These features make C-SPICE computationally attractive comparing to other existing grid based off-grid methods in which on-grid and off-grid related parameters are updated jointly and iteratively.

2. We show that C-SPICE is closely related to the Capon method [2] and SPICE [9], which is the origin of the name. Based on these observations, we obtain a simplified implementation of C-SPICE (SC-SPICE), whose complexity is insensitive to the grid size, for cases in which signals are independent and the number of snapshots is large.

3. In scenarios of coherent sources and small number of snapshots, we apply C-SPICE iteratively under the stochastic maximum likelihood (SML) criterion [32] and obtain an iterative C-SPICE algorithm (IC-SPICE). Our simulations show that the proposed methods provide a lower bound of root mean squared error (RMSE) in comparison with SPICE [9] with very attractive costs. It is also shown that the proposed methods can provide more accurate and faster DoA estimation than that of other existing grid-based methods, e.g., OGSBI-SVD [19]. Comparing to gridless methods, the proposed methods provide attractive complexity and performance tradeoff in all simulation scenarios. Also, they can be applied in different kinds of arrays, e.g., uniform circular array (UCA), for one-dimensional DoA estimation.

The following notations are adopted in this paper. The operators $(\cdot)^{T},(\cdot)^{H}$, and $(\cdot)^{\dagger}$ denote transpose, conjugate transpose, and Moore-Penrose inverse, respectively. Also, $\operatorname{diag}(\boldsymbol{x})$ returns a diagonal matrix with main diagonal $\boldsymbol{x}$. For an integer $N,[N]$ is defined as $\{1,2, \ldots, N\}$, and $\mathbb{C}^{N}$ represents the space of $N$-dimensional complex column vectors. $\|\boldsymbol{a}\|$ and $\|\boldsymbol{A}\|$ denote the $l_{2}$-norm and Frobenius norm of $\boldsymbol{a}$ and $\boldsymbol{A}$, respectively. $\mathbf{1}_{N}$ represents an $N \times 1$ vector with entries 1 and $\boldsymbol{I}$ an identity matrix. $\delta(k, l)$ denotes the Kronecker delta function. $\boldsymbol{R} \succeq 0$ and $\boldsymbol{R} \succ 0$ denote the matrix $\boldsymbol{R}$ is positive semidefinite and positive 
definite, respectively.

\section{Signal Model}

Consider the problem of estimating DoAs of $M$ far-field narrowband sources $s_{m}(t)$ located at $\theta_{m}, m \in[M]$, with an array of $N$ omnidirectional antennas. The model of array output can be written as [1]

$$
\boldsymbol{y}(t)=\sum_{m=1}^{M} \boldsymbol{a}\left(\theta_{m}\right) s_{m}(t)+\tilde{\boldsymbol{n}}(t), \quad t \in[T],
$$

where $\boldsymbol{y}(t)=\left[y_{1}(t), \ldots, y_{N}(t)\right]^{T}, \boldsymbol{a}\left(\theta_{m}\right)$ is the array steering vector of the $m$-th source, $\tilde{\boldsymbol{n}}(t)$ is the observation noise, and $T$ is the number of snapshots.

In the grid-based model, the DoA range, e.g., $\left(-\frac{\pi}{2}, \frac{\pi}{2}\right)$ for ULAs and $[-\pi, \pi)$ for UCAs, is sampled by a fix grid $\left\{\tilde{\theta}_{k}\right\}_{k=1}^{K}$ with $\tilde{\theta}_{k}-\tilde{\theta}_{k-1}=2 l_{\epsilon}$, where $K$ is grid size and $K \gg N>M$. Suppose that the true DoA $\theta_{m}$ is nearest to the grid point $\tilde{\theta}_{k_{m}}$ for some $k_{m} \in[K]$. Then we can approximate $\boldsymbol{a}\left(\theta_{m}\right)$ by its first order Taylor expansion [12]

$$
\boldsymbol{a}\left(\theta_{m}\right) \approx \boldsymbol{a}\left(\tilde{\theta}_{k_{m}}\right)+\boldsymbol{e}\left(\tilde{\theta}_{k_{m}}\right)\left(\theta_{m}-\tilde{\theta}_{k_{m}}\right),
$$

where $\boldsymbol{e}\left(\tilde{\theta}_{k_{m}}\right)$ denotes the first order derivative of $\boldsymbol{a}(\theta)$ at the point $\theta=\tilde{\theta}_{k_{m}}$. Defining $\epsilon_{k} \in\left[-l_{\epsilon}, l_{\epsilon}\right]$ and making use of (2), we can rewrite (1) as

$$
\boldsymbol{y}(t)=\sum_{k=1}^{K}\left[\boldsymbol{a}\left(\tilde{\theta}_{k}\right)+\boldsymbol{e}\left(\tilde{\theta}_{k}\right) \epsilon_{k}\right] x_{k}(t)+\boldsymbol{n}(t), t \in[T],
$$

where

$$
\begin{array}{lrl}
x_{k}(t)=s_{m}(t) \text { and } \epsilon_{k}=\theta_{m}-\tilde{\theta}_{k_{m}}, & \text { if } k=k_{m}, & \forall m \in[M], \\
x_{k}(t)=0, & \text { otherwise, }
\end{array}
$$

and $\boldsymbol{n}(t)$ is the summation of observation noise and errors induced by approxi- 
mation. Denoting

$$
\begin{aligned}
\boldsymbol{A} & =\left[\boldsymbol{a}\left(\tilde{\theta}_{1}\right), \boldsymbol{a}\left(\tilde{\theta}_{2}\right), \ldots, \boldsymbol{a}\left(\tilde{\theta}_{K}\right)\right], \\
\boldsymbol{E} & =\left[\boldsymbol{e}\left(\tilde{\theta}_{1}\right), \boldsymbol{e}\left(\tilde{\theta}_{2}\right), \ldots, \boldsymbol{e}\left(\tilde{\theta}_{K}\right)\right], \\
\boldsymbol{\Phi} & =\operatorname{diag}(\boldsymbol{\epsilon}), \boldsymbol{\epsilon}=\left[\epsilon_{1}, \epsilon_{2}, \ldots, \epsilon_{K}\right]^{T}, \\
\boldsymbol{x}(t) & =\left[x_{1}(t), x_{2}(t), \ldots, x_{K}(t)\right]^{T},
\end{aligned}
$$

we can rewrite (4) in a matrix form

$$
\boldsymbol{y}(t)=(\boldsymbol{A}+\boldsymbol{E} \boldsymbol{\Phi}) \boldsymbol{x}(t)+\boldsymbol{n}(t), t \in[T] .
$$

In the following derivation, we consider the noise to be spatially and temporally white Gaussian with equal variance $\sigma$ and uncorrelated with the signals. We also assume that the signals are uncorrelated with each other such that $E\left[s_{k}\left(t_{k}\right) s_{l}^{*}\left(t_{l}\right)\right]=p_{k} \delta(k, l) \delta\left(t_{k}, t_{l}\right)$ and the data snapshots have the following covariance matrix

$$
\begin{aligned}
\boldsymbol{R} & =E\left[\boldsymbol{y}(t) \boldsymbol{y}^{H}(t)\right] \\
& =\sum_{k=1}^{K} p_{k}\left(\boldsymbol{a}\left(\tilde{\theta}_{k}\right)+\boldsymbol{e}\left(\tilde{\theta}_{k}\right) \epsilon_{k}\right)\left(\boldsymbol{a}\left(\tilde{\theta}_{k}\right)+\boldsymbol{e}\left(\tilde{\theta}_{k}\right) \epsilon_{k}\right)^{H}+\sigma \boldsymbol{I} \\
& =[\boldsymbol{A}, \boldsymbol{E}, \boldsymbol{I}]\left[\begin{array}{lll}
\boldsymbol{I} & \boldsymbol{\Phi} & \mathbf{0} \\
\mathbf{0} & \mathbf{0} & \boldsymbol{I}
\end{array}\right]^{T} \boldsymbol{P}\left[\begin{array}{lll}
\boldsymbol{I} & \boldsymbol{\Phi} & \mathbf{0} \\
\mathbf{0} & \mathbf{0} & \boldsymbol{I}
\end{array}\right][\boldsymbol{A}, \boldsymbol{E}, \boldsymbol{I}]^{H} \\
& =\boldsymbol{B}^{H} \boldsymbol{D}^{H} \boldsymbol{P} \boldsymbol{D} \boldsymbol{B},
\end{aligned}
$$

where $p_{k}=E\left[\left\|s_{k}(t)\right\|^{2}\right]$ and

$$
\begin{aligned}
& \boldsymbol{B}=[\boldsymbol{A}, \boldsymbol{E}, \boldsymbol{I}]^{H}, \quad \boldsymbol{D}=\left[\begin{array}{lll}
\boldsymbol{I} & \boldsymbol{\Phi} & \mathbf{0} \\
\mathbf{0} & \mathbf{0} & \boldsymbol{I}
\end{array}\right], \\
& \boldsymbol{P}=\operatorname{diag}\left(\left[\boldsymbol{p}^{T}, \sigma \mathbf{1}_{N}^{T}\right]\right), \quad \boldsymbol{p}=\left[p_{1}, p_{2}, \ldots, p_{K}\right]^{T} .
\end{aligned}
$$

\section{The C-SPICE Algorithm}

In this section, we derive the sparse covariance-based off-grid DoA estimation method in two scenarios: the number of snapshot $T$ larger than the array size $N$ and its complement. 


\subsection{The Case of $T \geq N$}

When $T \geq N$, the inverse of both $\boldsymbol{R}$ and $\hat{\boldsymbol{R}}$ exist, where $\hat{\boldsymbol{R}}=\sum_{t=1}^{T} \boldsymbol{y}(t) \boldsymbol{y}^{H}(t) / T$. For the sake of parameter estimation, the following sparse covariance fitting criterion can be used [9]

$$
\begin{aligned}
\min _{\boldsymbol{p}, \sigma, \boldsymbol{\epsilon}} & f(\boldsymbol{p}, \sigma, \boldsymbol{\epsilon}) \\
\text { s.t. } & f(\boldsymbol{p}, \sigma, \boldsymbol{\epsilon})=\operatorname{tr}\left(\hat{\boldsymbol{R}} \boldsymbol{R}^{-1}\right)+\operatorname{tr}\left(\hat{\boldsymbol{R}}^{-1} \boldsymbol{R}\right), \\
& p_{k} \geq 0,\left|\epsilon_{k}\right| \leq l_{\epsilon}, \forall k \in[K], \\
& \sigma \geq 0 .
\end{aligned}
$$

Different from the optimization problem in SPICE [9, 33], problem (13) is nonlinear and nonconvex in general. Formal arguments for this property are given in Appendix A.

Proposition 1. Problem (13) is in general nonlinear and nonconvex.

To find the optimal solution of problem (13), we first introduce a new variable $C \in \mathbb{C}^{(2 K+N) \times N}$ and consider the following optimization problem

$$
\begin{aligned}
& \min _{\boldsymbol{C}} \operatorname{tr}\left(\boldsymbol{C}^{H} \boldsymbol{D}^{\dagger} \boldsymbol{P}^{-1} \boldsymbol{D}^{H \dagger} \boldsymbol{C}\right), \\
& \text { s.t. } \boldsymbol{B}^{H} \boldsymbol{C}=\hat{\boldsymbol{R}}^{1 / 2}
\end{aligned}
$$

where $\boldsymbol{D}^{\dagger}=\boldsymbol{D}^{H}\left(\boldsymbol{D} \boldsymbol{D}^{H}\right)^{-1}$. As the matrix $\boldsymbol{D}^{\dagger} \boldsymbol{P}^{-1} \boldsymbol{D}^{H \dagger}$ is rank deficient and positive semidefinite, the optima of (14) is not unique but one of them can be found by Proposition 2 .

Proposition 2. One optimal solution of problem (14) is given by

$$
\boldsymbol{C}^{*}=\boldsymbol{D}^{H} \boldsymbol{P} \boldsymbol{D} \boldsymbol{B} \boldsymbol{R}^{-1} \hat{\boldsymbol{R}}^{1 / 2}
$$

with the optimal objective value $\operatorname{tr}\left(\hat{\boldsymbol{R}} \boldsymbol{R}^{-1}\right)$, where $\boldsymbol{D}, \boldsymbol{B}, \boldsymbol{P}$, and $\boldsymbol{R}$ are defined in (11) and (12).

Proof. See Appendix B. 
Since the optimal value of problem (14) equals the first term of $f(\boldsymbol{p}, \sigma, \boldsymbol{\epsilon})$ in (13b), the optimal solution of problem (13) can be obtained by solving

$$
\begin{aligned}
\min _{\boldsymbol{p}, \sigma, \boldsymbol{\epsilon}, \boldsymbol{C}} & \operatorname{tr}\left(\boldsymbol{C}^{H} \boldsymbol{D}^{\dagger} \boldsymbol{P}^{-1} \boldsymbol{D}^{H \dagger} \boldsymbol{C}\right)+\operatorname{tr}\left(\hat{\boldsymbol{R}}^{-1} \boldsymbol{R}\right), \\
\text { s.t. } & \boldsymbol{B}^{H} \boldsymbol{C}=\hat{\boldsymbol{R}}^{1 / 2}, \\
& p_{k} \geq 0,\left|\epsilon_{k}\right| \leq l_{\epsilon}, \forall k \in[K], \\
& \sigma \geq 0
\end{aligned}
$$

Problem (16) is in the space of $(\boldsymbol{p}, \sigma, \boldsymbol{\epsilon}, \boldsymbol{C})$ and also nonconvex. However, this problem is easier to handle since optimizing part of the four variables while holding others fixed is trivial (e.g., closed form). We will exploit this property to design the C-SPICE algorithm.

First, the update of $\boldsymbol{C}$ is already derived by Proposition 2, see (15). Denoting $\boldsymbol{C}=\left[\boldsymbol{c}_{1}, \boldsymbol{c}_{2}, \ldots, \boldsymbol{c}_{2 K+N}\right]^{H}$ and substituting the definitions of $\boldsymbol{D}, \boldsymbol{B}$, and $\boldsymbol{P}$ into (15), we can rewrite (15) in vector form

$$
\begin{aligned}
\boldsymbol{c}_{k} & =p_{k} \hat{\boldsymbol{R}}^{1 / 2} \boldsymbol{R}^{-1}\left(\boldsymbol{a}\left(\tilde{\theta}_{k}\right)+\epsilon_{k} \boldsymbol{e}\left(\tilde{\theta}_{k}\right)\right), \\
\boldsymbol{c}_{K+k} & =\epsilon_{k} \boldsymbol{c}_{k}, \forall k \in[K], \\
{\left[\boldsymbol{c}_{2 K+1}, \ldots, \boldsymbol{c}_{2 K+N}\right] } & =\sigma \hat{\boldsymbol{R}}^{1 / 2} \boldsymbol{R}^{-1} .
\end{aligned}
$$

Second, update $\boldsymbol{p}, \sigma$, and $\boldsymbol{\epsilon}$ with fixed $\boldsymbol{C}$. Using the definitions of $\boldsymbol{P}, \boldsymbol{D}$, and $\boldsymbol{B}$ in (12) and the estimation of $\boldsymbol{C}$ in (17), the objective function of (16) can be equivalently transformed to

$$
f_{c}(\boldsymbol{p}, \sigma, \boldsymbol{\epsilon})=\sum_{k=1}^{K}\left(\frac{\left(1+\hat{\epsilon}_{k} \epsilon_{k}\right)^{2}\left\|\boldsymbol{c}_{k}\right\|^{2}}{\left(1+\epsilon_{k}^{2}\right)^{2} p_{k}}+p_{k}\left\|\tilde{\boldsymbol{a}}_{k}+\epsilon_{k} \tilde{\boldsymbol{e}}_{k}\right\|^{2}\right)+\frac{c_{\sigma}}{\sigma}+\sigma \operatorname{tr}\left(\hat{\boldsymbol{R}}^{-1}\right),
$$

where $c_{\sigma}=\sum_{k=2 K+1}^{2 K+N}\left\|\boldsymbol{c}_{k}\right\|^{2}, \hat{\epsilon}_{k}$ denotes the estimate of $\epsilon_{k}$ in the previous iteration, and

$$
\begin{gathered}
\tilde{\boldsymbol{a}}=\hat{\boldsymbol{R}}^{-1 / 2} \boldsymbol{a}, \\
\tilde{\boldsymbol{e}}=\hat{\boldsymbol{R}}^{-1 / 2} \boldsymbol{e} .
\end{gathered}
$$


Since $p_{k} \geq 0, \sigma \geq 0$, and $1+\hat{\epsilon}_{k} \epsilon_{k} \geq 0$ (by assuming $l_{\epsilon} \ll 1$ ), the inequality of arithmetic and geometric means can be used to show that

$$
f_{c}(\boldsymbol{p}, \sigma, \boldsymbol{\epsilon}) \geq 2 \sum_{k=1}^{K} \frac{\left(1+\hat{\epsilon}_{k} \epsilon_{k}\right)\left\|\boldsymbol{c}_{k}\right\|\left\|\tilde{\boldsymbol{a}}_{k}+\epsilon_{k} \tilde{\boldsymbol{e}}_{k}\right\|}{\left(1+\epsilon_{k}^{2}\right)}+2 \sqrt{c_{\sigma} \operatorname{tr}\left(\hat{\boldsymbol{R}}^{-1}\right)}
$$

where the equality holds if and only if

$$
\begin{aligned}
p_{k} & =\frac{\left(1+\hat{\epsilon}_{k} \epsilon_{k}\right)\left\|\boldsymbol{c}_{k}\right\|}{\left(1+\epsilon_{k}^{2}\right)\left\|\tilde{\boldsymbol{a}}_{k}+\epsilon_{k} \tilde{\boldsymbol{e}}_{k}\right\|}, k \in[K], \\
\sigma & =\sqrt{c_{\sigma} / \operatorname{tr}\left(\hat{\boldsymbol{R}}^{-1}\right)} .
\end{aligned}
$$

Therefore, $p_{k}$ and $\sigma$ can be updated according to (21), which is also in closed form.

Below we study the updating process of $\epsilon$. Substitute (21) into (18) to eliminate the variables $\boldsymbol{p}$ and $\sigma$. Then the objective function (18) becomes

$$
f_{c}(\boldsymbol{\epsilon})=\sum_{k=1}^{K} \frac{\left(1+\hat{\epsilon}_{k} \epsilon_{k}\right)\left\|\boldsymbol{c}_{k}\right\|\left\|\tilde{\boldsymbol{a}}_{k}+\epsilon_{k} \tilde{\boldsymbol{e}}_{k}\right\|}{\left(1+\epsilon_{k}^{2}\right)}+\sqrt{c_{\sigma} \operatorname{tr}\left(\hat{\boldsymbol{R}}^{-1}\right)} .
$$

The problem of minimizing $f_{c}(\boldsymbol{\epsilon})$ can be decoupled into $K$ subproblems, i.e., $\forall k \in[K]$,

$$
\begin{aligned}
& \min _{\epsilon_{k}} \frac{\left(1+\hat{\epsilon}_{k} \epsilon_{k}\right)\left\|\tilde{\boldsymbol{a}}_{k}+\epsilon_{k} \tilde{\boldsymbol{e}}_{k}\right\|}{1+\epsilon_{k}^{2}}, \\
& \text { s.t. }-l_{\epsilon} \leq \epsilon_{k} \leq l_{\epsilon} .
\end{aligned}
$$

It is obvious that problem (23) is nonconvex and solving $K$ such problems in each iteration is computationally expensive. Fortunately, when the grid is very fine and $l_{\epsilon} \ll 1$, we can assume that $\left(1+\hat{\epsilon}_{k} \epsilon_{k}\right) /\left(1+\epsilon_{k}^{2}\right)=1$, especially as the iteration converges. Therefore, we approximate the optimal solution of (23) by solving the following problem:

$$
\begin{gathered}
\min _{\epsilon_{k}}\left\|\tilde{\boldsymbol{a}}_{k}+\epsilon_{k} \tilde{\boldsymbol{e}}_{k}\right\| \\
\text { s.t. }-l_{\epsilon} \leq \epsilon_{k} \leq l_{\epsilon},
\end{gathered}
$$


whose solutions are in closed form, given by

$$
\epsilon_{k}^{*}=\left\{\begin{array}{cl}
-\frac{\operatorname{Re}\left\{\tilde{\boldsymbol{a}}_{k}^{H} \tilde{e}_{k}\right\}}{\left\|\tilde{e}_{k}\right\|^{2}}, & \text { if }\left|\frac{\operatorname{Re}\left\{\tilde{\boldsymbol{a}}_{k}^{H} \tilde{e}_{k}\right\}}{\left\|\tilde{e}_{k}\right\|^{2}}\right| \leq l_{\epsilon}, \\
-l_{\epsilon}, & \text { if }-\frac{\operatorname{Re}\left\{\tilde{\boldsymbol{a}}_{k}^{H} \tilde{\boldsymbol{e}}_{k}\right\}}{\left\|\tilde{e}_{k}\right\|^{2}} \leq-l_{\epsilon}, \\
l_{\epsilon}, & \text { if }-\frac{\operatorname{Re}\left\{\tilde{\boldsymbol{a}}_{k}^{H} \tilde{\boldsymbol{e}}_{k}\right\}}{\left\|\tilde{\boldsymbol{e}}_{k}\right\|^{2}} \geq l_{\epsilon} .
\end{array}\right.
$$

Finally, the algorithm can be initialized with the least squares estimates based on (14b) which are given as follows:

$$
\begin{aligned}
\boldsymbol{c}_{k} & =\arg \min _{\boldsymbol{c}}\left\|\left(\boldsymbol{a}\left(\tilde{\theta}_{k}\right)+\hat{\epsilon}_{k} \boldsymbol{e}\left(\tilde{\theta}_{k}\right)\right) \boldsymbol{c}^{H}-\hat{\boldsymbol{R}}^{1 / 2}\right\| \\
& =\frac{1}{\left\|\boldsymbol{a}\left(\tilde{\theta}_{k}\right)+\hat{\epsilon}_{k} \boldsymbol{e}\left(\tilde{\theta}_{k}\right)\right\|^{2}} \hat{\boldsymbol{R}}^{1 / 2}\left(\boldsymbol{a}\left(\tilde{\theta}_{k}\right)+\hat{\epsilon}_{k} \boldsymbol{e}\left(\tilde{\theta}_{k}\right)\right), k \in[K], \\
{\left[\boldsymbol{c}_{2 K+1}, \ldots, \boldsymbol{c}_{2 K+N}\right] } & =\arg \min _{\boldsymbol{c}_{2 K+1}, \ldots, \boldsymbol{c}_{2 K+N}}\left\|\left[\boldsymbol{c}_{2 K+1}, \ldots, \boldsymbol{c}_{2 K+N}\right]-\hat{\boldsymbol{R}}^{1 / 2}\right\| \\
& =\hat{\boldsymbol{R}}^{1 / 2} .
\end{aligned}
$$

We summarize C-SPICE in Table 1 , where $f_{c}(i)$ denotes the value of $f_{c}(\boldsymbol{\epsilon})$ in the $i$-th iteration.

Table 1: The C-SPICE algorithm for $T>N$.

\section{Initialize:}

1.1 Fix $\delta_{1}, I_{1}$. Let $i=0$.

1.2 Estimate $\boldsymbol{\epsilon}$ according to (25),

1.3 Initialize $\boldsymbol{C}$ according to (26),

2. Repeat: $i=i+1$,

2.1 Update $\boldsymbol{p}$ and $\sigma$ using (21),

2.2 Update $\boldsymbol{R}$ using (11),

2.3 Update $\boldsymbol{C}$ using (17),

3. Terminate: if $\left(f_{c}(i-1)-f_{c}(i)\right) / f_{c}(i)<\delta_{1}$ or $i>I_{1}$.

4. Estimation: Denote the index of the largest $M$ peaks in the spectrum of $\boldsymbol{p}$ as $\left\{k_{1}, \cdots, k_{M}\right\}$, then the m-th DoA estimate is $\tilde{\theta}_{k_{m}}+\epsilon_{k_{m}}^{*}$.

Remark 1. In Table 1, $\boldsymbol{\epsilon}$ is estimated only once and it is independent of the on-grid parameters, i.e., $\boldsymbol{p}$ and $\sigma$. Note that when $\boldsymbol{\epsilon}$ is given, (13) is convex 
to $\boldsymbol{p}$ and $\sigma$. Thus the iterations in C-SPICE can converge to a global optimal solution (dependent on $\boldsymbol{\epsilon}$ ) from any initial point.

\subsection{The Case of $T<N$}

When $T<N$, the inverse of $\hat{\boldsymbol{R}}$ does not exist and we cannot use (24) to estimate $\boldsymbol{\epsilon}$. To overcome this, we resort to the SML criterion [32] and obtain the optimization problem

$$
\begin{aligned}
\min _{\boldsymbol{p}, \sigma, \boldsymbol{\epsilon}} & f_{L}(\boldsymbol{p}, \sigma, \boldsymbol{\epsilon}) \\
\text { s.t. } & f_{L}(\boldsymbol{p}, \sigma, \boldsymbol{\epsilon})=\ln |\boldsymbol{R}|+\operatorname{tr}\left(\boldsymbol{R}^{-1} \hat{\boldsymbol{R}}\right), \\
& p_{k} \geq 0,\left|\epsilon_{k}\right| \leq l_{\epsilon}, \forall k \in[K], \\
& \sigma \geq 0 .
\end{aligned}
$$

Since $\ln |\boldsymbol{R}|$ is nonconvex in $(\boldsymbol{p}, \sigma, \boldsymbol{\epsilon}),(27)$ is hard to solve. We resort to a majorization minimization approach to solve the problem approximately. Recall that the function $\ln |\boldsymbol{R}|$ is concave in $\boldsymbol{R}, \forall \boldsymbol{R} \succ 0$ [34]. According to the firstorder condition for concave functions [34], we have

$$
\ln |\boldsymbol{R}| \leq \ln \left|\boldsymbol{R}_{0}\right|+\operatorname{tr}\left(\boldsymbol{R}_{0}^{-1} \boldsymbol{R}\right)-N, \quad \forall \boldsymbol{R} \succ 0, \quad \forall \boldsymbol{R}_{0} \succ 0,
$$

and the equation holds when $\boldsymbol{R}=\boldsymbol{R}_{0}$. According to (11), $\boldsymbol{R}$ and $\boldsymbol{R}_{0}$ in problem (27) can be denoted by $\boldsymbol{R}(\boldsymbol{p}, \sigma, \boldsymbol{\epsilon})$ and $\boldsymbol{R}\left(\boldsymbol{p}_{0}, \sigma_{0}, \boldsymbol{\epsilon}_{0}\right)$, respectively, with $(\boldsymbol{p}, \sigma, \boldsymbol{\epsilon})$ and $\left(\boldsymbol{p}_{0}, \sigma_{0}, \boldsymbol{\epsilon}_{0}\right)$ being in the feasible set of (27). It should be mentioned that when $\sigma=0$ (or $\sigma_{0}=0$ ), there may be $\boldsymbol{R} \succeq 0$ (or $\boldsymbol{R}_{0} \succeq 0$ ), which is different from the constraints in (28). Since $\sigma>0$ is always satisfied in practice, this problem can be readily circumvented by modifying constraint (27d) to $\sigma \geq \kappa$ with $\kappa$ being a small positive number.

Defining

$$
g(\boldsymbol{p}, \sigma, \boldsymbol{\epsilon}) \triangleq \ln \left|\boldsymbol{R}_{0}\right|+\operatorname{tr}\left(\boldsymbol{R}_{0}^{-1} \boldsymbol{R}\right)-N+\operatorname{tr}\left(\boldsymbol{R}^{-1} \hat{\boldsymbol{R}}\right),
$$

and making use of (28), we can obtain that for any $(\boldsymbol{p}, \sigma, \boldsymbol{\epsilon})$ and $\left(\boldsymbol{p}_{0}, \sigma_{0}, \boldsymbol{\epsilon}_{0}\right)$ in the feasible set of problem (27), there is

$$
f_{L}(\boldsymbol{p}, \sigma, \boldsymbol{\epsilon}) \leq g(\boldsymbol{p}, \sigma, \boldsymbol{\epsilon}),
$$


and the equation holds when $(\boldsymbol{p}, \sigma, \boldsymbol{\epsilon})=\left(\boldsymbol{p}_{0}, \sigma_{0}, \boldsymbol{\epsilon}_{0}\right)$. Denote $\left(\boldsymbol{p}_{1}, \sigma_{1}, \boldsymbol{\epsilon}_{1}\right)$ as the minimum point of $g(\boldsymbol{p}, \sigma, \boldsymbol{\epsilon})$. It follows from (30) that

$$
f_{L}\left(\boldsymbol{p}_{0}, \sigma_{0}, \boldsymbol{\epsilon}_{0}\right)=g\left(\boldsymbol{p}_{0}, \sigma_{0}, \boldsymbol{\epsilon}_{0}\right) \geq g\left(\boldsymbol{p}_{1}, \sigma_{1}, \boldsymbol{\epsilon}_{1}\right) \geq f_{L}\left(\boldsymbol{p}_{1}, \sigma_{1}, \boldsymbol{\epsilon}_{1}\right)
$$

According to (31), we can approach a local optimal solution of (27) by successively solving the optimization problem

$$
\begin{aligned}
\min _{\boldsymbol{p}, \sigma, \boldsymbol{\epsilon}} & \operatorname{tr}\left(\boldsymbol{R}_{0}^{-1} \boldsymbol{R}\right)+\operatorname{tr}\left(\boldsymbol{R}^{-1} \hat{\boldsymbol{R}}\right) \\
\text { s.t. } & p_{k} \geq 0,\left|\epsilon_{k}\right| \leq l_{\epsilon}, \forall k \in[K], \\
& \sigma \geq 0
\end{aligned}
$$

where $\boldsymbol{R}_{0}$ is constructed according to (11) with $(\boldsymbol{p}, \sigma, \boldsymbol{\epsilon})$ obtained from the previous iteration. Since $\boldsymbol{R}_{0}$ is invertible, problem (32) is similar to problem (13). As a result, C-SPICE proposed in Section 3.1 can be used. To initialize $\boldsymbol{R}_{0}$, we set $\boldsymbol{\epsilon}=\mathbf{0}$ and estimate $\boldsymbol{p}$ and $\sigma$ based on the sparse covariance fitting criterion for the $T<N$ case, which is

$$
\min _{\boldsymbol{p} \geq 0, \sigma \geq 0}\left\|\boldsymbol{R}^{-1 / 2}(\hat{\boldsymbol{R}}-\boldsymbol{R})\right\|^{2}
$$

Problem (33) can be efficiently solved by SPICE [33]. The procedure of the algorithm for $T<N$ is summarized in Table 2, where $f_{L}(i)$ denotes the value of $f_{L}(\boldsymbol{p}, \sigma, \boldsymbol{\epsilon})$ in the i-th iteration.

Remark 2. We refer to the algorithm in Table 2 as IC-SPICE. If (32) can be solved optimally in each step, then $f_{L}(i)$ decreases monotonically with $i$ according to (31) and IC-SPICE converges to a local optima. C-SPICE is however not guaranteed to obtain a global optimal solution of (32). In this case, we continue to update $\boldsymbol{p}, \sigma$, and $\boldsymbol{\epsilon}$ through iterations and use the group that minimizes $f_{L}(\boldsymbol{p}, \sigma, \boldsymbol{\epsilon})$ to generate the DoA estimates.

\section{Connections to Prior Works}

\subsection{Connections to SPICE and SPA}

The C-SPICE method proposed in Section 3.1 is closely related to SPICE in [33] and SPA in [11]. In general, the three methods are all based on the 
Table 2: The Algorithm for $T<N$.

\section{Initialize:}

1.1 Fix $I_{2}$. Let $i=0$ and $\boldsymbol{\epsilon}=\mathbf{0}$.

1.2 Estimate $\boldsymbol{p}$ and $\sigma$ by solving problem (33) via SPICE [33],

1.3 Construct $\boldsymbol{R}_{0}$ according to (11).

2. Repeat: $i=i+1$,

$2.1 \boldsymbol{p}, \sigma$, and $\boldsymbol{\epsilon}$ are updated by solving problem (32) via C-SPICE,

$2.2 \boldsymbol{R}_{0}$ is updated by (11).

3. Terminate: if $i=I_{2}$.

4. Estimation: Calculate $\epsilon$ according to (25) and estimate DoAs

based on $\boldsymbol{p}$ and $\boldsymbol{\epsilon}$.

same covariance fitting criteria, but their abilities in modeling the true signal are different. Specifically, SPA can model the signal precisely. On the contrary, SPICE uses a discretized sampling grid to approximate continuous spatial spectrum. This approximation may cause inaccurate DoA estimation for three reasons: the modeling mismatch, the on-grid issue, and the identifiability problem [11]. In C-SPICE, the former two errors are alleviated by the additional vector $\boldsymbol{\epsilon}$, which models deviation between the grid and the true DoA. The identifiability problem appears when the signal to noise ratio (SNR) is low such that the estimated $\boldsymbol{p}$ is not sparse. While this problem can be alleviated by using the postprocessing procedure in [11], C-SPICE in Table 1 uses a simple heuristic method, i.e., taking the maximum peaks in the spectrum of $\boldsymbol{p}$ as the DoA estimates, such that the performance improvement induced by off-grid model and those by postprocessing can be distinguished.

The computational complexity of SPICE is $\mathcal{O}\left(N^{3} K I_{r}\right)$, where $I_{r} \leq I_{1}$ denotes the number of iterations. Compared with SPICE, the additional cost of C-SPICE comes from calculating $\boldsymbol{\epsilon}$, which is $\mathcal{O}\left(N^{3} K\right)$. The computational complexity of SPA is $\mathcal{O}\left(N^{6.5}\right)$ [11]. Apparently, C-SPICE can provide an effective tradeoff between the modeling accuracy and complexity. 


\subsection{Connection to the Capon Method}

Note that the off-grid error $\epsilon_{k_{m}}^{*}$ in (25) is obtained by solving (24), whose objective function is an approximation of the second order Taylor expansion of the Capon spectrum [2], i.e.,

$$
\begin{aligned}
P_{c a p}(\theta) & =\boldsymbol{a}^{H}(\theta) \hat{\boldsymbol{R}}^{-1} \boldsymbol{a}(\theta) \\
& =\left\|\tilde{\boldsymbol{a}}_{k}+\epsilon_{k} \tilde{\boldsymbol{e}}_{k}\right\|^{2}+\operatorname{Re}\left(\boldsymbol{a}_{k}^{H} \hat{\boldsymbol{R}}^{-1} \boldsymbol{g}_{k}\right) \epsilon_{k}^{2}+\mathcal{O}\left(\epsilon_{k}^{3}\right) \\
& \approx\left\|\tilde{\boldsymbol{a}}_{k}+\epsilon_{k} \tilde{\boldsymbol{e}}_{k}\right\|^{2}, \quad\left|\theta-\theta_{k}\right|<l_{\epsilon} \ll 1,
\end{aligned}
$$

where $\epsilon_{k}=\theta-\theta_{k}, \operatorname{Re}(\cdot)$ denotes the real part of a variable, and $\boldsymbol{g}_{k}$ the second order derivative of $\boldsymbol{a}(\theta)$ at $\theta_{k}$. Therefore, C-SPICE is the abbreviation of Capon-SPICE, where SPICE provides the global vision of the spectrum of $\boldsymbol{p}$ and the approximation of Capon spectrum the local information. This simple observation makes C-SPICE distinct from other existing grid-based off-grid DoA estimation methods. More specifically, the methods in existing literature, e.g., $[14,15,16,17,18,19]$, require to update the on-grid and off-grid related unknown parameters jointly, while the off-grid error $\epsilon$ in C-SPICE is estimated independent of $\boldsymbol{p}$ and $\sigma$ in a closed form. The advantage of this decoupling is that C-SPICE can be implemented with a much higher efficiency. Moreover, C-SPICE in Table 1 can be further simplified by using the local information provided by $\epsilon$. In particular, suppose that the SNR and the grid size are large enough and $\tilde{\theta}_{k_{m}}$ is the grid nearest to the true DoA $\theta_{m}$, then the optimal solution of (24a) (without the bound constraint (24b)) must satisfy $\left|\epsilon_{k_{m}}^{*}\right| \approx\left|\tilde{\theta}_{k_{m}}-\theta_{m}\right| \leq l_{\epsilon}$. In other words, if $\left|\epsilon_{k}^{*}\right|>l_{\epsilon}$, then $\tilde{\theta}_{k}$ is not a grid closest to any true DoA. As a result, we can set $p_{k}=0$ and $\boldsymbol{c}_{k}=\mathbf{0}$ in step 2 of Table 1 whenever $\left|\epsilon_{k}^{*}\right|>l_{\epsilon}$, which leads to a simplified version of C-SPICE (SC-SPICE). On the other hand, at the cost of higher efficiency, C-SPICE cannot estimate DoAs of coherent signals, which is also a limitation of the Capon method. In this case, we apply IC-SPICE in Table 2, based on the SML criterion.

\subsection{Connection to LIKES}


IC-SPICE is closely related to the likelihood-based estimation of sparse parameters method (LIKES) [33]. In general, LIKES and IC-SPICE are both based on the SML criterion and solve the SML problem by iteratively applying SPICE and C-SPICE, respectively. However, LIKES does not consider the offgrid error $\boldsymbol{\epsilon}$, which makes the two algorithms being different from one another. First, LIKES is an on-grid method, while IC-SPICE is an off-grid algorithm. Second, IC-SPICE is essential when $T<N$ or coherent signal exists since CSPICE does not work in this case, whereas LIKES is to improve the estimation accuracy of SPICE.

\section{Simulation Results}

Here, we present simulation results to evaluate the proposed algorithms. The following algorithms are compared: SPICE [33], LIKES [33], OGSBI-SVD [19], SPA [11], ANM [25], TVMA with an SVD front end (TVMA-FAST) [30], Unitary ESPRIT [6], Capon [2], C-SPICE in Section 3.1, SC-SPICE in Section 4.2, and IC-SPICE in Section 3.2. SPICE and LIKES are on-grid model based methods, OGSBI-SVD is a grid-based off-grid method, SPA, ANM, and TVMAFAST are gridless sparse methods. ${ }^{1}$ When $T>N$, ANM applies a dimension reduction operation as in [24]. OGSBI-SVD and TVMA-FAST are implemented as in [19] and [30], respectively. Unitary ESPRIT follows from Table I in [6] with maximum overlap. The parameters for C-SPICE in Table 1 and IC-SPICE in Table 2 are chosen as $I_{1}=1000, I_{2}=1$, and $\delta_{1}=10^{-3}$. We consider DoA estimation with ULAs in the first four simulations and with a UCA and an irregular array in the others.

In Experiment 1, we compare C-SPICE with the other methods in terms of RMSE and computational time with respect to the SNR. We consider $M=3$ independent equal power signal sources from directions $\frac{180^{\circ}}{\pi}\left[-\frac{1}{3}, \frac{1}{13}, \frac{1}{4}\right]$. A ULA

\footnotetext{
${ }^{1}$ The Matlab codes of SPICE, LIKES, OGSBI-SVD, SPA, and ANM are all available online at https://www.it.uu.se/katalog/davza513 and https://sites.google.com/site/zaiyang0248/publication, respectively.
} 
with $N=16$ is used to receive the signal. The number of snapshots is chosen as $T=100$. The RMSE is defined as

$$
\sqrt{\frac{1}{M J} \sum_{m=1}^{M} \sum_{j=1}^{J}\left\|\theta_{m}-\hat{\theta}_{m}(j)\right\|^{2}},
$$

where $J=300$ is the number of independent trials and $\hat{\theta}_{m}(j)$ denotes the estimate of $\theta_{m}$ in the $j$-th trial. The simulation results are presented in Fig. 1. In this figure, the numbers 200,500 , and 1000 in the legend denote the RMSE curves obtained by using the grid $\left\{\tilde{\theta}_{k}\right\}_{k=1}^{K}$ with $K=200, K=500$, and $K=1000$, respectively. The Cramer-Rao bound (CRB) is also shown as a performance benchmark. Fig. 1a illustrates the RMSE of the compared methods. It is seen that SPICE with a finer sampling space has a smaller RMSE bound, which is dependent on the distances between the true DoAs and the grid. OGSBI-SVD performs better than SPICE, and its RMSEs with $K=200$ and $K=500$ are lower bounded when SNR is larger than $10 \mathrm{~dB}$ and $25 \mathrm{~dB}$, respectively. The RMSE of C-SPICE with $K=200$ is lower bounded when SNR $\geq 25 \mathrm{~dB}$ and the one with $K=500$ decreases linearly with the SNR and approaches CRB. It is obvious that C-SPICE is the most robust to off-grid DoAs in the three grid-based methods. Also, ANM, Capon, and SPA perform similarly to C-SPICE with $K=500$. The performance of C-SPICE and SCSPICE are comparable. A gap about 4dB exists between the RMSE results of Unitary ESPRIT (marked as "UEsprit") and CRB.

The average running times corresponding to the above RMSE curves are plotted in Fig. 1b. It is seen that the running time of C-SPICE is less than that of SPICE, although the former needs to estimate a new variable $\epsilon$. The reason is that the grid mismatch is first calibrated by the estimate of $\boldsymbol{\epsilon}$ in C-SPICE, which makes it converge faster in estimating $\boldsymbol{p}$ and $\sigma$. We can also see that SC-SPICE is faster than the Capon method, but slower than Unitary Esprit. Note that we choose the grid size $K=30000$ for the Capon method such that its RMSE coincides with the CRB in the tested SNR range. According to the results in the two figures, C-SPICE with $K=500$ achieves the CRB in the 


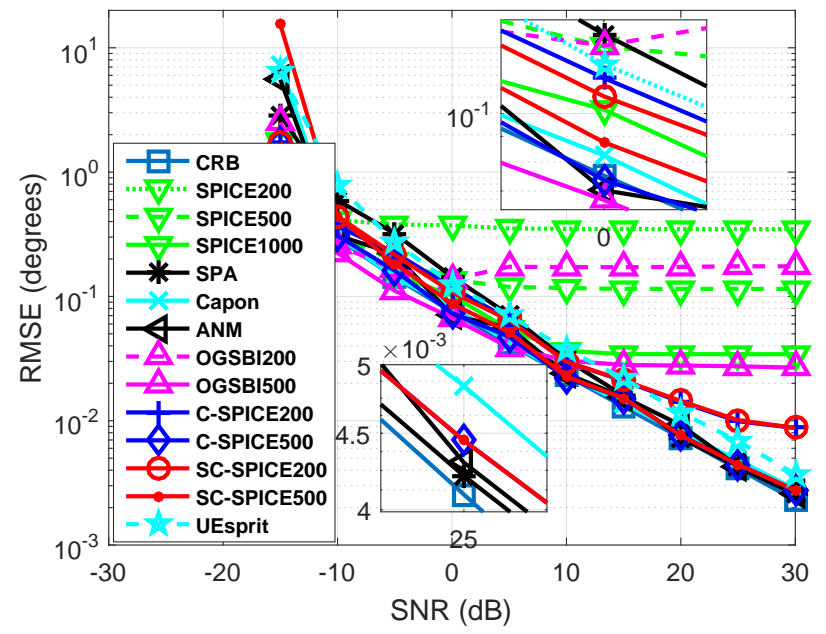

(a)

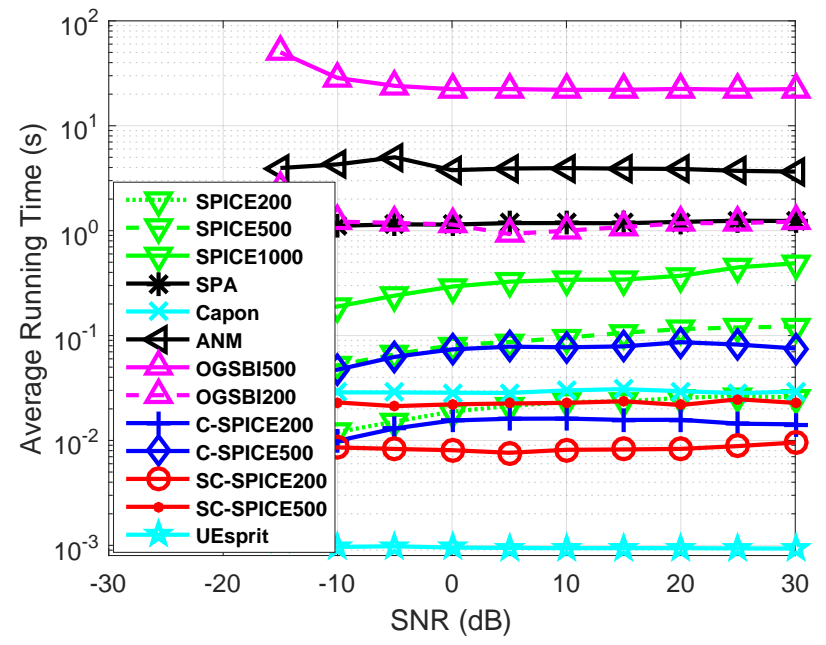

(b)

Figure 1: Performance comparison of different methods with a ULA, $N=16$, and $T=100$. (a) RMSE versus SNR, (b) average running time versus SNR.

tested SNR range with an average running time about $\frac{1}{15}, \frac{1}{50}$, and $\frac{1}{500}$ of those of SPA, ANM, and OGSBI-SVD, respectively. The running time of SC-SPICE is about $\frac{1}{3}$ of that of C-SPICE. We will not consider OGSBI-SVD with $K=500$ 
in the later experiments due to its high complexity.

Experiment 2 investigates the RMSE performance of IC-SPICE when coherent signals exist or $T<N$. We repeat Experiment 1 but set $T=5$ and $T=200$, respectively. When $T=200$, the first two sources are coherent with each other with correlation coefficient $\rho=1$. The RMSE curves with $T=5$ and $T=200$ are plotted in Fig. 2a and Fig. 2b, respectively. When $T=5$, IC-SPICE and ANM can approach the CRB in the tested SNR range, the RMSEs of OGSBI-SVD, SPICE, and LIKES are all lower bounded, and a large gap exists between the curves of SPA and CRB. The corresponding average running times of IC-SPICE with $K=200$ and $K=500$ are $0.07 \mathrm{~s}$ and $0.37 \mathrm{~s}$, respectively, and those of SPICE and LIKES with $K=500$, SPA, ANM, and OGSBI-SVD with $K=200$ are $0.25 \mathrm{~s}, 0.37 \mathrm{~s}, 3 \mathrm{~s}, 2 \mathrm{~s}$, and $1.6 \mathrm{~s}$, respectively. When $T=200$ and coherent signals exist, it is seen in Fig. $2 \mathrm{~b}$ that the RMSEs of IC-SPICE and OGSBI-SVD are lower bounded at SNR $\geq 30 \mathrm{~dB}$ and SNR $\geq 10 \mathrm{~dB}$, respectively, the gaps between the CRB and the RMSE curves of SPA and ANM are increased to more than $10 \mathrm{~dB}$, and that of the Unitary ESPRIT escalates to more than $20 \mathrm{~dB}$. We can also see that LIKES is more accurate than SPICE in the low SNR regime. However, they share the same RMSE lower bound as SNR increases; only IC-SPICE can attain the CRB. Note that at the points of $0 \mathrm{~dB}$ to $10 \mathrm{~dB}$, the RMSE of SPA is contaminated by the frequency splitting phenomenon [35].

Experiment 3 studies the performance variation with respect to the number of antennas $N$. In the simulations, three equal power signal sources are located at $\frac{180^{\circ}}{\pi}\left[-\frac{1}{3}, \frac{1}{13}, \frac{1}{2}\right]$. The number of snapshots and SNR are set to $T=5$ and $20 \mathrm{~dB}$, respectively. The simulation results are presented in Fig. 3. We can see that a large gap exists between the RMSE of SPA and the CRB yet the running time of SPA increases exponentially with $N$. The RMSEs of SPICE, OGSBI-SVD with $K=200$, and IC-SPICE with $K=200$ are lower bounded. IC-SPICE with $K=500$ can achieve the CRB with an average running time about $1 / 4$ of that of ANM.

In Experiment 4, the resolution of the proposed methods is investigated via 


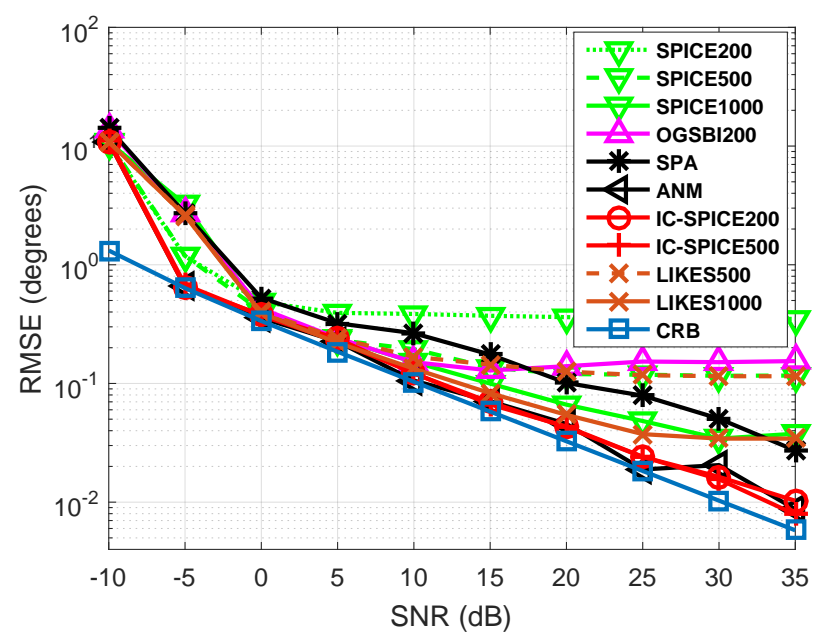

(a)

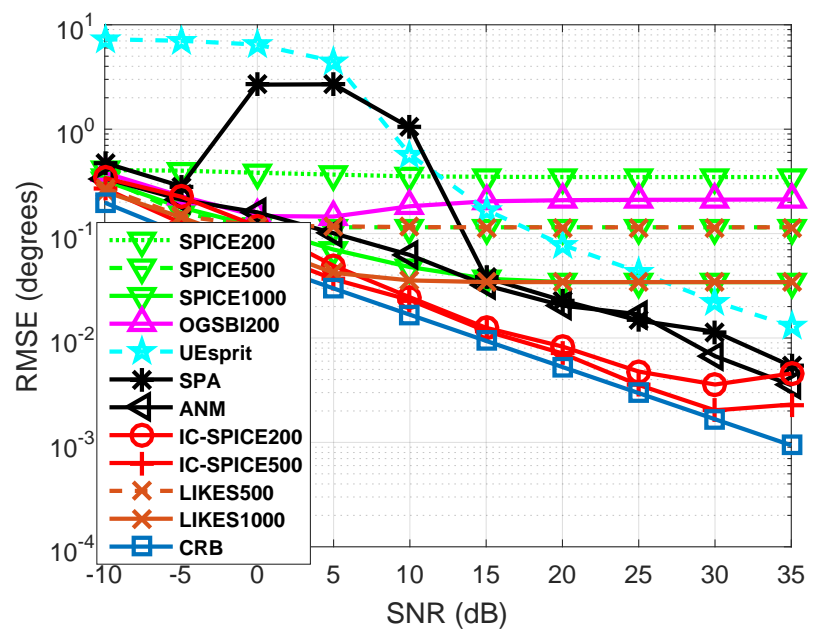

(b)

Figure 2: RMSE of DoA estimates of IC-SPICE compared with some existing methods and the CRB with $N=16$. (a) $T=5$ and independent sources, (b) $T=200$ and the first two sources are coherent.

bias. As resolving closely spaced sources is a hard task, especially at low SNRs, small bias can be considered as a good compromise, if the peaks can be resolved [8]. We now study the biases of different algorithms by estimating DoAs of two 


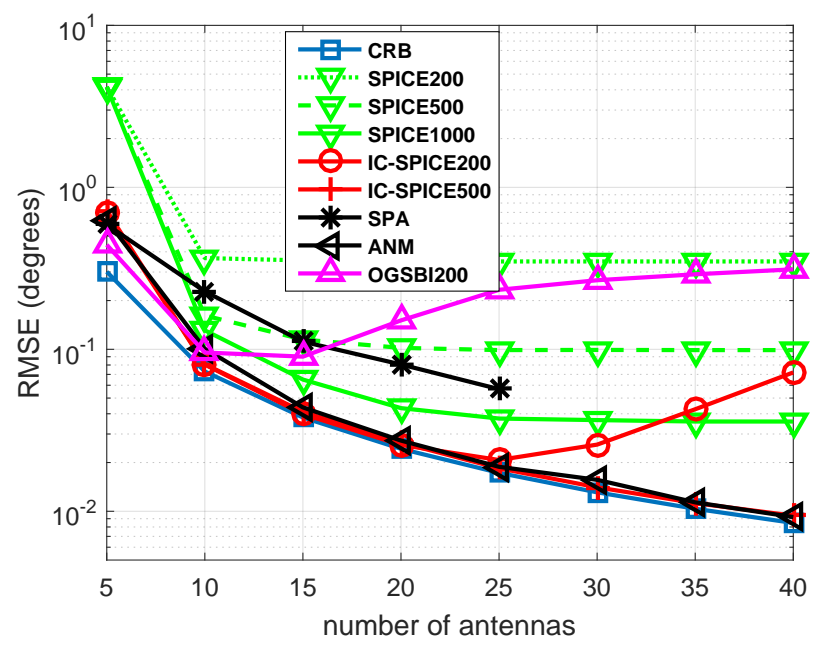

(a)

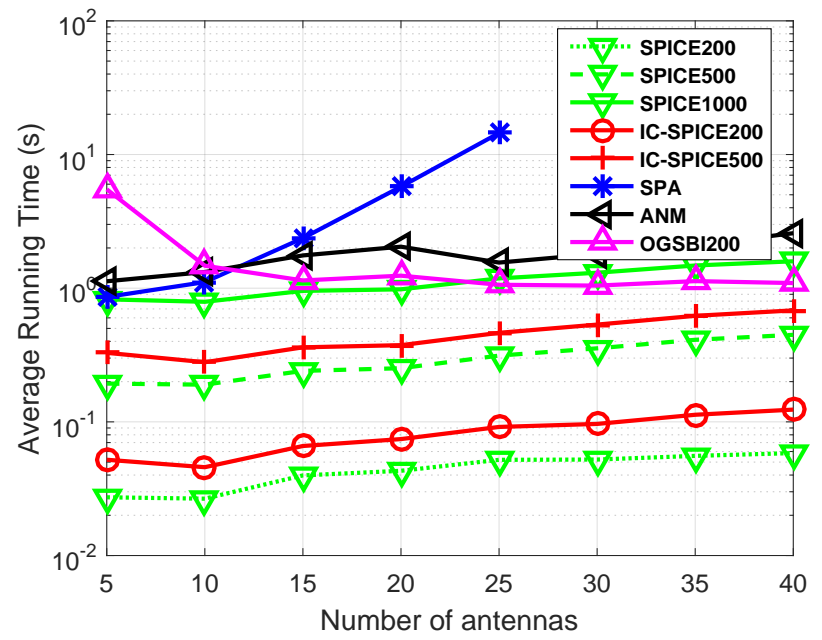

(b)

Figure 3: Performance comparison of different methods with $N=16$ and $T=5$. (a) RMSE against $\mathrm{N}$, (b) average running time against $\mathrm{N}$.

independent sources and varying the angular separation between them. The SNR is set as $30 \mathrm{~dB}$. The numbers of sensors, snapshots, and independent trials are $N=16, T=5$, and $J=1000$, respectively. Fig. 4 illustrates the biases of 


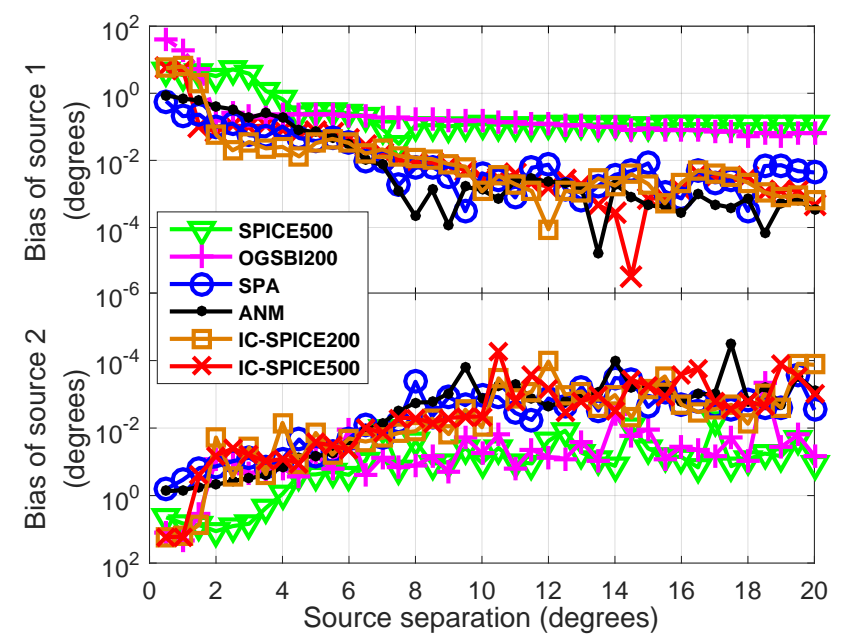

Figure 4: Biases of different methods in estimation of two sources against separation between the two sources with SNR $=30 \mathrm{~dB}$

each of the two DoA estimates against the angular separation when one source is fixed at $-10^{\circ}$ and the other varies from $-9.5^{\circ}$ to $10^{\circ}$. We can see the presence of biases for low separations in all the methods, the biases of SPA, ANM, and IC-SPICE are decreased to less than $10^{-2}$ when the sources are more than $7^{\circ}$ apart, while the biases of the other methods are lower bounded by $10^{-2}$.

Experiment 5 studies the RMSE performance of DoA estimation with a UCA. The considered UCA contains 12 antennas and is impinged by three equal power signals from directions $\frac{180^{\circ}}{\pi}\left[-\frac{1}{2}, \frac{1}{13}, \frac{2}{3}\right]$. Two different scenarios are considered: first, the number of snapshots is $T=200$ and the signals are independent; second, $T=5$ and the first two signals are coherent with each other. Fig. 5a plots simulation results of the first scenario. We can see that RMSEs of all the grid-based methods are lower bounded, the RMSE bound of C-SPICE is lower than those of SPICE and OGSBI-SVD with the same grid, and the RMSE of C-SPICE with $K=200$ is lower than that of Capon with gird size $K=30000$. In the figure, the RMSE curve of TVMA-FAST approaches CRB when SNR varies from $-5 \mathrm{~dB}$ to $0 \mathrm{~dB}$, but a gap appears between the two and increases to about $7 \mathrm{~dB}$ as SNR increases. The gap may be induced 
by approximating the infinite series of Jacobi-Anger expansion by a finite series during the derivation of TVMA. The average running times of SPICE and CSPICE are similar, which are 0.03s, 0.14s, and 0.61s with grid sizes 200, 500, and 1000, respectively, and those of OGSBI-SVD are 1.54s and 46.79s with grid sizes 200 and 500, respectively. The running time of SC-SPICE is insensitive to the grid size and equals 0.015s, which is lower than Capon's 0.022s and TVMAFAST's 1.35s. The simulation results of the second scenario are shown in Fig. 5b. We observe that IC-SPICE performs better than the other methods. The gap between the RMSE of IC-SPICE and the CRB can be reduced by increasing $I_{2}$ and decreasing $\delta_{1}$, simultaneously. The average running times of IC-SPICE with $K=200$ and $K=500$ are 0.074 s and $0.46 \mathrm{~s}$, respectively, and that of OGSBI-SVD with $K=200$ is 2.04 s.

In experiment 6, we demonstrate the utility of C-SPICE when the sensor arrangement is irregular. The array configuration is shown in Fig. 6, where $N=$ 12 sensors are randomly located with in the region between two concentric circles with radii $5 \lambda$ and $6.5 \lambda$ with $\lambda$ denoting the wavelength of working frequency. We assume that the array is illuminated by two uncorrelated sources, whose elevation angles are $90^{\circ}$ and azimuth angles are $\theta_{1}$ and $\theta_{2}$ with $\theta_{1}$ uniformly generated in between $\left[0,360^{\circ}\right)$ and $\theta_{2}=\theta_{1}+10^{\circ}$. The number of snapshots is chosen as $T=100$. The RMSE performance of DoA estimation based on 300 independent Monte Carlo runs is shown in Fig. 7. The findings are similar to those in Fig. 5a. The average running times are about 10s for TVMA-FAST and less than $0.6 \mathrm{~s}$ for the other methods.

\section{Conclusions}

This paper studied the off-grid DoA estimation problem to compensate for the modeling errors induced by discretizing the continuous range of the DoA. We applied the off-grid DoA model to the sparse covariance fitting criterion and derived a DoA estimation algorithm. For scenarios of coherent signals and small number of snapshots, an algorithm based on the SML criterion was derived. 


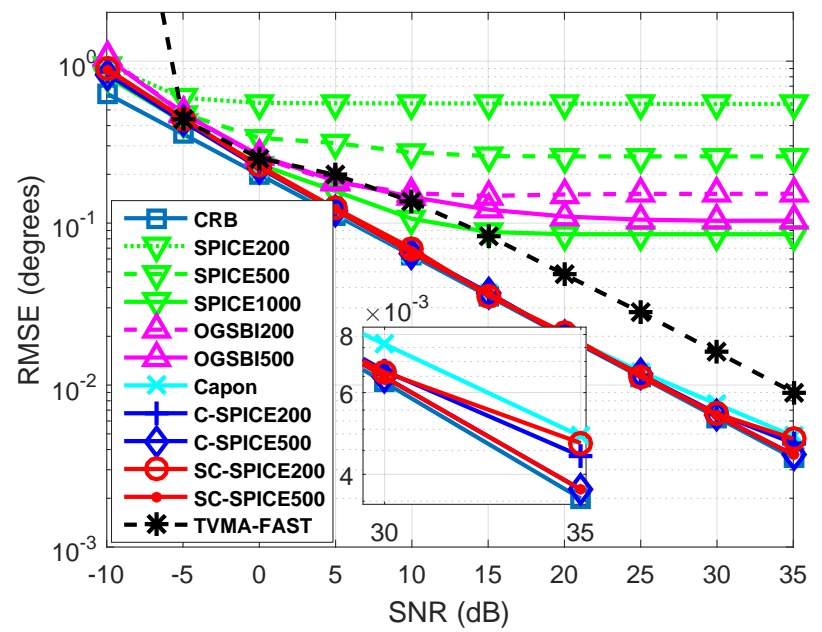

(a)

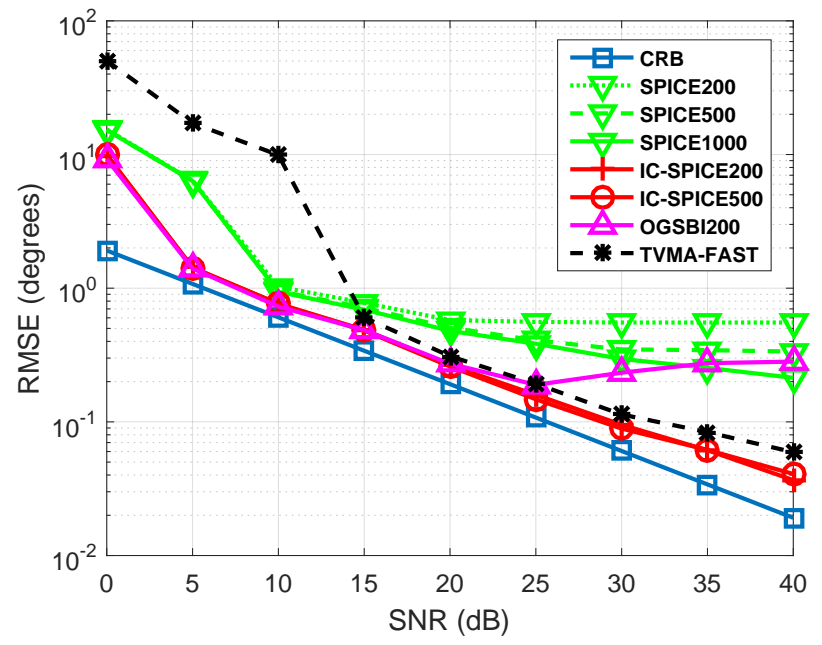

(b)

Figure 5: RMSE against SNR with a UCA, $N=12$, and three sources. (a) $T=200$ and independent sources, (b) $T=5$ and the first two sources are coherent.

Experiments were performed to demonstrate that the proposed methods can provide an accurate and fast DoA estimation. 


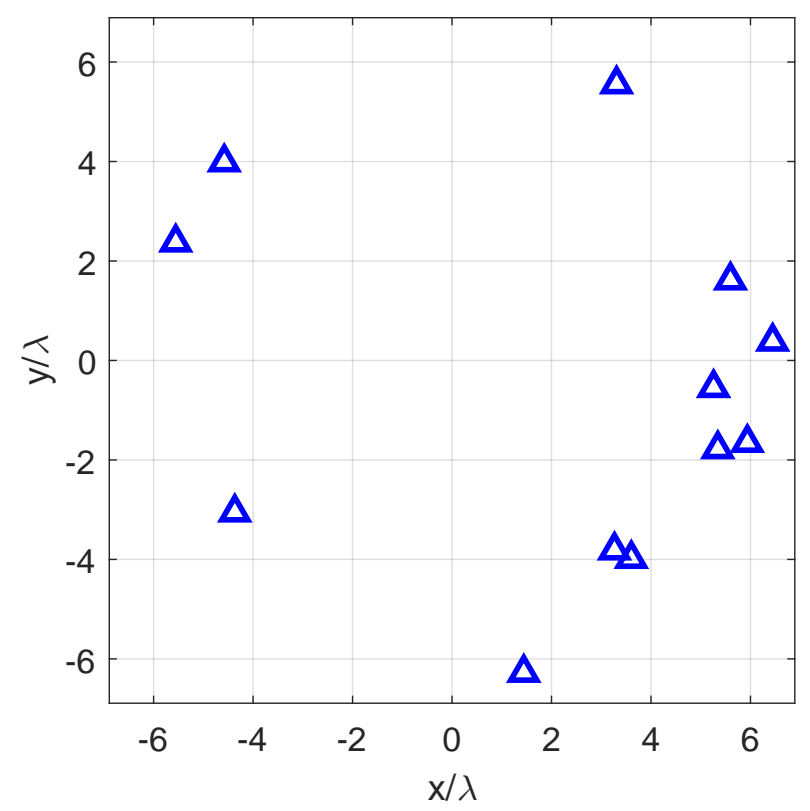

Figure 6: Array arrangement for experiment 6

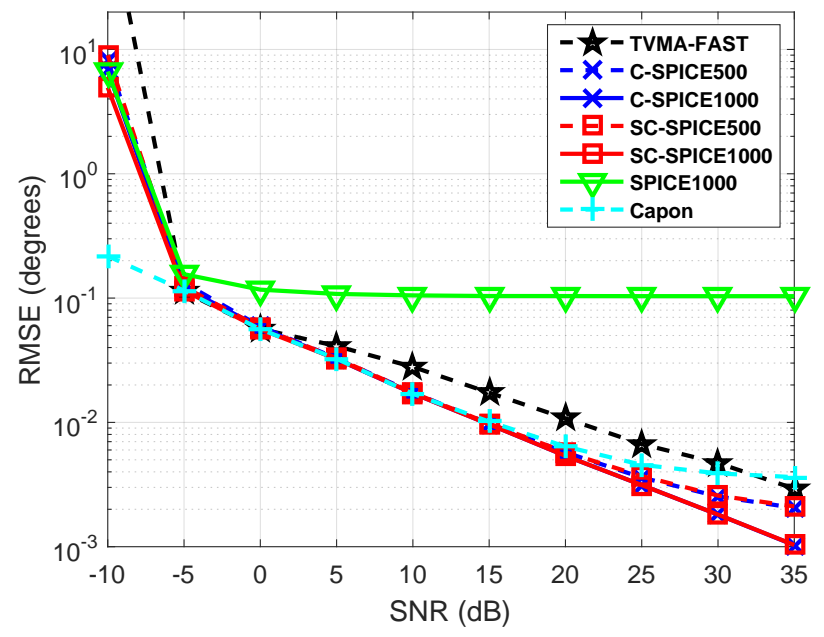

Figure 7: RMSE against SNR with the array arrangement in Fig. 6 and $T=100$. 


\section{Appendix A. Proof of the Proposition 1}

According to the definition of convex optimization problem [34], problem (13) is convex only if its objective function $f(\boldsymbol{p}, \sigma, \boldsymbol{\epsilon})$ is. The latter is further equivalent to the Hessian matrix $\nabla^{2} f(\boldsymbol{p}, \sigma, \boldsymbol{\epsilon}) \succeq 0$ for all $(\boldsymbol{p}, \sigma, \boldsymbol{\epsilon}) \in \operatorname{dom} f$, where $\operatorname{dom} f$ denotes the feasible set of (13). Moreover, since $\nabla^{2} f(\boldsymbol{p}, \sigma, \boldsymbol{\epsilon})$ is symmetric, it is positive semidefinite iff all of its principal minors are nonnegative [36]. Therefore, we only need to find one point in $\operatorname{dom} f$ such that some principal minor is negative.

W.L.O.G., consider one principal minor of $\nabla^{2} f(\boldsymbol{p}, \sigma, \boldsymbol{\epsilon}),|\boldsymbol{G}|$, where $|\boldsymbol{G}|$ denotes determinant of matrix $\boldsymbol{G}$ and

$$
\boldsymbol{G}=\left[\begin{array}{cc}
\frac{\partial^{2} f}{\partial p_{1}^{2}} & \frac{\partial^{2} f}{\partial p_{1} \partial \epsilon_{1}} \\
\frac{\partial^{2} f}{\partial \epsilon_{1} \partial p_{1}} & \frac{\partial^{2} f}{\partial \epsilon_{1}^{2}}
\end{array}\right]
$$

Now let us calculate $|\boldsymbol{G}|$. For easy of expression, denote $\boldsymbol{a}=\boldsymbol{a}\left(\tilde{\theta}_{1}\right), \boldsymbol{e}=$ $\boldsymbol{e}\left(\tilde{\theta}_{1}\right), p=p_{1}$, and $\epsilon=\epsilon_{1}$. Then

$$
\begin{aligned}
\boldsymbol{R} & =\sum_{k=1}^{K} p_{k}\left(\boldsymbol{a}\left(\tilde{\theta}_{k}\right)+\boldsymbol{e}\left(\tilde{\theta}_{k}\right) \epsilon_{k}\right)\left(\boldsymbol{a}\left(\tilde{\theta}_{k}\right)+\boldsymbol{e}\left(\tilde{\theta}_{k}\right) \epsilon_{k}\right)^{H}+\sigma \boldsymbol{I} \\
& =p(\boldsymbol{a}+\boldsymbol{e} \epsilon)(\boldsymbol{a}+\boldsymbol{e} \epsilon)^{H}+\boldsymbol{T}
\end{aligned}
$$

where $\boldsymbol{T}=\sum_{k=2}^{K} p_{k}\left(\boldsymbol{a}\left(\tilde{\theta}_{k}\right)+\boldsymbol{e}\left(\tilde{\theta}_{k}\right) \epsilon_{k}\right)\left(\boldsymbol{a}\left(\tilde{\theta}_{k}\right)+\boldsymbol{e}\left(\tilde{\theta}_{k}\right) \epsilon_{k}\right)^{H}+\sigma \boldsymbol{I}$. When $\sigma>0$ (which is always satisfied in practical DoA estimation problem), $\boldsymbol{T}$ and $\boldsymbol{R}$ are invertible. Using matrix inversion lemma, we have

$$
\boldsymbol{R}^{-1}=\boldsymbol{T}^{-1}-\boldsymbol{T}^{-1} p(\boldsymbol{a}+\boldsymbol{e} \epsilon)\left(1+(\boldsymbol{a}+\boldsymbol{e} \epsilon)^{H} \boldsymbol{T}^{-1} p(\boldsymbol{a}+\boldsymbol{e} \epsilon)\right)^{-1}(\boldsymbol{a}+\boldsymbol{e} \epsilon)^{H} \boldsymbol{T}^{-1} .
$$

Substituting $\boldsymbol{R}$ and $\boldsymbol{R}^{-1}$ into $f(\boldsymbol{p}, \sigma, \boldsymbol{\epsilon})$ leads to

$$
\begin{aligned}
f(\boldsymbol{p}, \sigma, \boldsymbol{\epsilon})= & p(\boldsymbol{a}+\boldsymbol{e} \epsilon)^{H} \hat{\boldsymbol{R}}^{-1}(\boldsymbol{a}+\boldsymbol{e} \epsilon)-\frac{p(\boldsymbol{a}+\boldsymbol{e} \epsilon)^{H} \boldsymbol{T}^{-1} \hat{\boldsymbol{R}} \boldsymbol{T}^{-1}(\boldsymbol{a}+\boldsymbol{e} \epsilon)}{1+p(\boldsymbol{a}+\boldsymbol{e} \epsilon)^{H} \boldsymbol{T}^{-1}(\boldsymbol{a}+\boldsymbol{e} \epsilon)} \\
& +\operatorname{tr}\left(\hat{\boldsymbol{R}} \boldsymbol{T}^{-1}\right)+\operatorname{tr}\left(\hat{\boldsymbol{R}}^{-1} \boldsymbol{T}\right) .
\end{aligned}
$$


Defining

$$
\begin{aligned}
k_{1} & =\boldsymbol{a}^{H} \hat{\boldsymbol{R}}^{-1} \boldsymbol{a}, k_{2}=\boldsymbol{a}^{H} \hat{\boldsymbol{R}}^{-1} \boldsymbol{e}, k_{3}=\boldsymbol{e}^{H} \hat{\boldsymbol{R}}^{-1} \boldsymbol{e}, \\
m_{1} & =\boldsymbol{a}^{H} \boldsymbol{T}^{-1} \boldsymbol{a}, m_{2}=\boldsymbol{a}^{H} \boldsymbol{T}^{-1} \boldsymbol{e}, m_{3}=\boldsymbol{e}^{H} \boldsymbol{T}^{-1} \boldsymbol{e}, \\
n_{1} & =\boldsymbol{a}^{H} \boldsymbol{T}^{-1} \hat{\boldsymbol{R}} \boldsymbol{T}^{-1} \boldsymbol{a}, n_{2}=\boldsymbol{a}^{H} \boldsymbol{T}^{-1} \hat{\boldsymbol{R}} \boldsymbol{T}^{-1} \boldsymbol{e}, n_{3}=\boldsymbol{e}^{H} \boldsymbol{T}^{-1} \hat{\boldsymbol{R}} \boldsymbol{T}^{-1} \boldsymbol{e},
\end{aligned}
$$

and substituting them into (A.4), we obtain

$$
\begin{aligned}
f(\boldsymbol{p}, \sigma, \boldsymbol{\epsilon})= & p\left(k_{1}+\epsilon k_{2}+\epsilon k_{2}^{*}+\epsilon^{2} k_{3}\right)-\frac{p\left(n_{1}+\epsilon n_{2}+\epsilon n_{2}^{*}+\epsilon^{2} n_{3}\right)}{1+p\left(m_{1}+\epsilon m_{2}+\epsilon m_{2}^{*}+\epsilon^{2} m_{3}\right)} \\
& +\operatorname{tr}\left(\hat{\boldsymbol{R}} \boldsymbol{T}^{-1}\right)+\operatorname{tr}\left(\hat{\boldsymbol{R}}^{-1} \boldsymbol{T}\right),
\end{aligned}
$$

where $k^{*}$ denotes the complex conjugate of $k$.

Calculate $\frac{\partial^{2} f}{\partial p_{1}^{2}}, \frac{\partial^{2} f}{\partial p_{1} \partial \epsilon_{1}}, \frac{\partial^{2} f}{\partial \epsilon_{1}^{2}}$, and then $|\boldsymbol{G}|$ based on (A.6) (by using the Symbolic Math Toolbox in Matlab2015b). We can obtain that when $\epsilon=0$ there is

$$
|\boldsymbol{G}|_{\epsilon=0, p}=\sum_{i=0}^{5} c_{i} p^{i},
$$

where $c_{i}, i=1,2, \ldots, 5$, only depend on $k_{l}, m_{l}$, and $n_{l}, l=1,2,3, c_{5}=$ $-\frac{m_{1}^{5}\left(k_{2}+k_{2}^{*}\right)^{2}}{\left(m_{1} p+1\right)^{5}}$, and $c_{0}=-\frac{\left(k_{2}-n_{2}+k_{2}^{*}-n_{2}^{*}\right)^{2}}{\left(m_{1} p+1\right)^{5}}$. Since $m_{1}>0, p \geq 0$, and $k_{2}+k_{2}^{*} \neq 0$ (or $k_{2}-n_{2}+k_{2}^{*}-n_{2}^{*} \neq 0$ ) in general, we then have $c_{0}<0$ (or $c_{5}<0$ ). Therefore, $|\boldsymbol{G}|_{\epsilon=0, p}<0$ when $p$ is very large (or approaches 0 ) according to (A.7). In summary, when $\sigma>0, \epsilon_{1}=0$, and $p_{1}(>0)$ is large (or small) enough, there is $|\boldsymbol{G}|<0$ and hence, $\nabla^{2} f(\boldsymbol{p}, \sigma, \boldsymbol{\epsilon}) \succeq 0$ is not satisfied. Since $\epsilon_{1}=0$, $\sigma>0$, and $p_{1}>0$ satisfy the constraints in (13), we conclude that $f(\boldsymbol{p}, \sigma, \boldsymbol{\epsilon})$ is nonconvex.

\section{Appendix B. Proof of the Proposition 2}

The proof contains two steps. We first demonstrate that one optimal solution of (14) is in the column space of $\boldsymbol{D}^{H}$. Assume that $\boldsymbol{C}_{0}$ is any given point in the optimal solution set of (14) and define $\boldsymbol{C}_{1}=\boldsymbol{P}_{\boldsymbol{D}^{H}} \boldsymbol{C}_{0}$, where $\boldsymbol{P}_{\boldsymbol{D}^{H}}=\boldsymbol{D}^{\dagger} \boldsymbol{D}$ 
is the projection matrix to the column space of $\boldsymbol{D}^{H}$. Substituting the identity $\boldsymbol{D}^{\dagger}=\boldsymbol{P}_{\boldsymbol{D}^{H}} \boldsymbol{D}^{\dagger}$ into (14a), we have

$$
\begin{aligned}
& \operatorname{tr}\left(\boldsymbol{C}_{0}^{H} \boldsymbol{D}^{\dagger} \boldsymbol{P}^{-1} \boldsymbol{D}^{H \dagger} \boldsymbol{C}_{0}\right) \\
= & \operatorname{tr}\left(\boldsymbol{C}_{0}^{H} \boldsymbol{P}_{\boldsymbol{D}^{H}} \boldsymbol{D}^{\dagger} \boldsymbol{P}^{-1} \boldsymbol{D}^{H \dagger} \boldsymbol{P}_{\boldsymbol{D}^{H}} \boldsymbol{C}_{0}\right) \\
= & \operatorname{tr}\left(\boldsymbol{C}_{1}^{H} \boldsymbol{D}^{\dagger} \boldsymbol{P}^{-1} \boldsymbol{D}^{H \dagger} \boldsymbol{C}_{1}\right) .
\end{aligned}
$$

Therefore, $\boldsymbol{C}_{1}$ is an optimal solution of (14) and $\boldsymbol{C}_{1}=\boldsymbol{P}_{\boldsymbol{D}^{H}} \boldsymbol{C}_{0}=\boldsymbol{D}^{H} \boldsymbol{G}$, $\boldsymbol{G} \in \mathbb{C}^{(K+N) \times N}$.

Second, we show that under the constraints (14b) and $\boldsymbol{C}=\boldsymbol{D}^{H} \boldsymbol{G}$,

$$
\operatorname{tr}\left(\boldsymbol{C}^{H} \boldsymbol{D}^{\dagger} \boldsymbol{P}^{-1} \boldsymbol{D}^{H \dagger} \boldsymbol{C}\right) \geq \operatorname{tr}\left(\hat{\boldsymbol{R}} \boldsymbol{R}^{-1}\right) .
$$

Inserting both constraints into (B.2) leads to

$$
\operatorname{tr}\left(\boldsymbol{G}^{H} \boldsymbol{P}^{-1} \boldsymbol{G}\right) \geq \operatorname{tr}\left(\boldsymbol{G}^{H} \boldsymbol{D} \boldsymbol{B} \boldsymbol{R}^{-1} \boldsymbol{B}^{H} \boldsymbol{D}^{H} \boldsymbol{G}\right), \forall \boldsymbol{G} \in \mathbb{C}^{(K+N) \times N} .
$$

The inequality (B.3) holds if and only if $\boldsymbol{P}^{-1}-\boldsymbol{D} \boldsymbol{B} \boldsymbol{R}^{-1} \boldsymbol{B}^{H} \boldsymbol{D}^{H}$ is positive semidefinite. According to the Schur complement condition [34], the positive semidefinite condition is further equivalent to

$$
\left[\begin{array}{cc}
\boldsymbol{P}^{-1} & \boldsymbol{D} \boldsymbol{B} \\
\boldsymbol{B}^{H} \boldsymbol{D}^{H} & \boldsymbol{R}
\end{array}\right]=\left[\begin{array}{c}
\boldsymbol{P}^{-1 / 2} \\
\boldsymbol{B}^{H} \boldsymbol{D}^{H} \boldsymbol{P}^{1 / 2}
\end{array}\right]\left[\begin{array}{ll}
\boldsymbol{P}^{-1 / 2} & \boldsymbol{P}^{1 / 2} \boldsymbol{D} \boldsymbol{B}
\end{array}\right] \succeq 0 .
$$

Therefore, the inequality (B.2) holds. Moreover, it can be readily verified that the equality in (B.2) holds when $\boldsymbol{C}=\boldsymbol{C}^{*}$.

\section{References}

[1] H. Krim, M. Viberg, Two decades of array signal processing research: the parametric approach, IEEE Signal Process. Mag. 13 (4) (1996) 67-94.

[2] J. Capon, High-resolution frequency-wavenumber spectrum analysis, Proc. IEEE 57 (8) (1969) 1408-1418.

[3] R. Schmidt, A signal subspace approach to multiple emitter location spectral estimation, Ph.d. dissertation, Stanford Univ., Stanford, CA (1981). 
[4] R. Roy, T. Kailath, Esprit-estimation of signal parameters via rotational invariance techniques, IEEE Trans. Acoust., Speech Signal Process. 37 (7) (1989) 984-995.

[5] M. L. McCloud, L. L. Scharf, A new subspace identification algorithm for high-resolution doa estimation, IEEE Trans. Antennas Propag. 50 (10) (2002) 1382-1390.

[6] M. Haardt, J. A. Nossek, Unitary esprit: how to obtain increased estimation accuracy with a reduced computational burden, IEEE Transactions on Signal Processing 43 (5) (1995) 1232-1242.

[7] J. Fuchs, On the application of global matched filter to doa estimation with uniform circular arrays, IEEE Trans. Signal Process. 47 (4) (1999) 187-200.

[8] D. Malioutov, M. Cetin, A. Willsky, A sparse signal reconsctrution perspective for source localization with sensor arrays, IEEE Trans. Signal Process. 53 (8) (2005) 3010-3022.

[9] P. Stoica, P. Babu, J. Li, Spice: A sparse covariance-based estimation method for array processing, IEEE Trans. Signal Process. 59 (2) (2011) 629-638.

[10] M. Carlin, P. Rocca, G. Oliveri, F. Viani, A. Massa, Directions-of-arrival estimation through bayesian compressive sensing strategies, IEEE Trans. Antennas Propag. 61 (7) (2013) 3828-3838.

[11] Z. Yang, L. Xie, C. Zhang, A discretization-free sparse and parametric approach for linear array signal processing, IEEE Transactions on Signal Processing 62 (19) (2014) 4959-4973.

[12] H. Zhu, G. Leus, G. B. Giannakis, Sparsity-cognizant total least-squares for perturbed compressive sampling, IEEE Trans. Signal Process. 59 (5) (2011) 2002-2016. 
[13] J. Zheng, M. Kaveh, Directions-of-arrival estimation using a sparse spatial spectrum model with uncertainty, in: Proc. IEEE ICASSP, 2011, pp. 28482851.

[14] H. Abeida, Q. Zhang, J. Li, N. Merabtine, Iterative sparse asymptotic minimum variance based approaches for array processing, IEEE Trans. Signal Process. 61 (4) (2013) 933-944.

[15] T. Huang, Y. Liu, H. Meng, X. Wang, Adaptive matching pursuit with constrained total least squares, EURASIP J. Adv. Signal Process. 2012 (1) (2012) 76 .

URL http://dx.doi.org/10.1186/1687-6180-2012-76

[16] Z. Yang, C. Zhang, L. Xie, Robustly stable signal recovery in compressed sensing with structured matrix perturbation, IEEE Trans. Signal Process. 60 (9) (2011) 4658-4671.

[17] L. Hu, Z. Shi, J. Zhou, Q. Fu, Compressed sensing of complex sinusoids: An approach based on dictionary refinement, IEEE Trans. Signal Process. 60 (7) (2012) 3809-3822.

[18] M. F. Duarte, R. G. Baraniuk, Spectral compressive sensing, Appl. Comput. Harmon. Anal. 35 (1) (2013) 111-129.

[19] Z. Yang, L. Xie, C. Zhang, Off-grid direction of arrival estimation using sparse bayesian inference, IEEE Trans. Signal Process. 61 (1) (2013) 3843.

[20] E. J. Candès, C. Fernandez-Granda, Towards a mathematical theory of super-resolution, Commun. Pure Appl. Math. 67 (6) (2014) 906-956.

[21] P. Stoica, R. Moses, Spectral analysis of signals, Pearson/Prentice Hall Upper Saddle River, NJ, 2005.

[22] G. Tang, B. N. Bhaskar, P. Shah, B. Recht, Compressed sensing off the grid, IEEE Trans. Inf. Theory 59 (11) (2013) 7465-7490. 
[23] Z. Yang, L. Xie, Continuous compressed sensing with a single or multiple measurement vectors, in: Proc. IEEE Workshop Statist. Signal Process. (SSP), 2014, pp. 288-291.

[24] Z. Yang, L. Xie, Enhancing sparsity and resolution via reweighted atomic norm minimization, IEEE Trans. Signal Process. 64 (4) (2016) 995-1006.

[25] Z. Yang, L. Xie, Exact joint sparse frequency recovery via optimization methods, IEEE Trans. Signal Process. 64 (19) (2016) 5145-5157.

[26] B. N. Bhaskar, B. Recht, Atomic norm denoising with applications to line spectral estimation, IEEE Trans. Signal Process. 61 (23) (2012) 261-268.

[27] E. J. Candès, C. Fernandez-Granda, Super-resolution from noisy data, J. Fourier Anal. Appl. 19 (6) (2012) 1229-1254.

[28] Y. Chen, Y. Chi, Robust spectral compressed sensing via structured matrix completion, IEEE Trans. Inf. Theory 60 (10) (2014) 6576-6601.

[29] V. Chandrasekaran, B. Recht, P. A. Parrilo, A. S. Willsky, The convex geometry of linear inverse problems, Found. Comput. Math. 12 (6) (2012) 805-849.

[30] K. Mahata, M. M. Hyder, Grid-less t.v minimization for doa estimation, Signal Processing 132 (2017) 155-164.

[31] B. Ottersten, P. Stoica, R. Roy, Covariance matching estimation techniques for array signal processing applications, Digit. Signal Process. 8 (3) (1998) $185-210$.

[32] A. G. Jaffer, Maximum likelihood direction finding of stochastic sources: a separable solution, in: Proc. IEEE ICASSP, Vol. 5, 1988, pp. 2893-2896.

[33] P. Stoica, P. Babu, Spice and likes: Two hyperparameter-free methods for sparse-parameter estimation, Signal Process. 92 (7) (2012) 1580-1590. 
[34] S. Boyd, L. Vandenberghe, Convex Optimization, Cambridge Univ. Press, 2009.

[35] Z. Yang, L. Xie, On gridless sparse methods for line spectral estimation from complete and incomplete data, IEEE Trans. Signal Process. 63 (12) (2015) 3139-3153.

[36] C. D. Meyer, Matrix Analysis and Applied Linear Algebra, SIAM, 2001. 